\title{
Dispositifs acousto-optiques dispersifs programmables pour lasers ultrabrefs
}

Présents dès les premiers systèmes laser impulsionnels, les composants acousto-optiques fournissent des fonctionnalités essentielles aux systèmes ultrabrefs actuels et futurs.

Quelles sont ces propriétés ? Pourquoi l'acousto-optique ? Cet article présente quelques dispositifs actuellement présents dans les systèmes femtosecondes et détaille le dispositif de façonnage d'amplitude et de phase spectrales Dazzler.

\author{
$>$ Thomas OKSENHENDLER \\ FASTLITE \\ thoksen@fastlite.com
}

\section{L'intérêt des dispositifs acousto-optiques}

Dans la course à la brièveté des impulsions lasers et de leurs puissances crêtes, les lasers ont évolué pour passer du régime continu aux régimes $\mathrm{Q}$-switchés, mode-lockés et à l'amplification par impulsion à dérive de fréquence (Chirped Pulse Amplification).

Ces différentes étapes ont nécessité l'utilisation de modulateurs optiques commutant rapidement l'amplitude, modifiant la fréquence optique ou modifiant l'amplitude et la phase spectrales.

\section{Réseaux de Bragg élasto-optiques}

Les propriétés photoacoustiques ou élasto-optiques de certains cristaux permettent de créer des réseaux de Bragg par la modification des indices optiques du matériau par une onde acoustique. Ce principe permet, selon les cristaux et coupes cristallines choisis, de diffracter I'onde lumineuse incidente vers I'onde diffractée avec des variations possibles de l'angle de diffraction, de la modulation d'amplitude ou de phase spectrale, et de la fréquence optique.

L'onde acoustique est générée dans le cristal par le biais d'un transducteur piézoélectrique (généralement du Niobate de Lithium - $\mathrm{LiNbO}_{3}$ ). Ce transducteur transforme la tension électrique qui lui est appliquée en une déformation mécanique. Collé au cristal par une fine couche de métal, le transducteur transmet cette déformation au cristal. Une tension sinusoïdale crée alors une onde acoustique de fréquence pure se propageant dans le cristal. La déformation de la maille cristalline par cette onde acoustique entraîne une modification des indices optiques du cristal, traduite par les coefficients élasto-optiques, créant un réseau d'indice. C'est ce réseau qui diffracte l'onde incidente (figure 1).

Cette possibilité de créer un réseau d'indice de façon programmable est amplifiée par la richesse de la faune de type $d^{\prime}$ 'onde acoustique (longitudinale, transverse ou de surface) et par la flore des caractéristiques optiques des cristaux (isotrope, anisotrope uniaxe ou biaxe). Sans entrer dans une description exhaustive des dispositifs existants ou possibles (voir par exemples les livres AcoustoOptic Devices de J. Xu et R. Stroud ou Design and Fabrication of Acousto-optic Devices de A.P. Goutzoulis et D.R. Pape), nous allons illustrer les possibilités de cette technologie par quelques exemples de modulation optique.

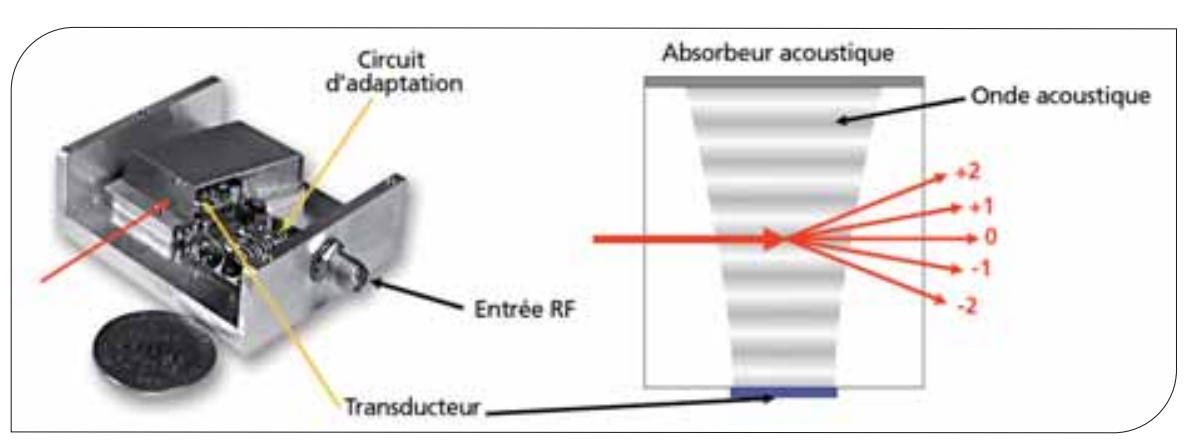

Figure 1. Dispositif acousto-optique.

\section{Modulateur acousto-optique}

Un modulateur acousto-optique (MAO) ou cellule de Bragg diffracte une partie de l'onde incidente sur une onde diffractée dont les propriétés dépendent à la fois de l'onde incidente et de la diffraction. Les caractéristiques de la diffraction sont fixées par la modulation d'indice optique générée par l'onde acoustique, elle-même générée par une onde radio-fréquence (de quelques $\mathrm{MHz}$ au $\mathrm{GHz}$ ). La seule différence avec un réseau de Bragg fixe est le fait que l'onde acoustique se propage dans le cristal, ce qui permet de la modifier.

Comme avec les réseaux fixes, il est possible de défléchir la lumière, de moduler son intensité, son amplitude, sa phase ou sa polarisation. Le réseau se propageant dans le cristal, il est également possible d'utiliser l'effet Doppler pour modifier la fréquence optique de l'onde diffractée.

\section{Déflecteur acousto-optique}

La déflection de la lumière par un déflecteur acousto-optique s'obtient simplement par la modulation du pas du réseau via la fréquence acoustique. En effet, la loi des réseaux impose $\sin (\theta)=(m \lambda / 2 \Lambda)$, 
avec $\theta$ l'angle de diffraction, $m=\ldots-2$, $-1,0,1,2, \ldots$ l'ordre de diffraction, $\lambda$ la longueur d'onde optique et $\Lambda=V / F_{a c}$, longueur d'onde acoustique, inversement proportionnel à la fréquence acoustique $\mathrm{F}_{\mathrm{ac}}$ car la vitesse acoustique $\mathrm{V}$ est constante sur une large gamme de fréquence. L'angle de déflection peut ainsi être modulé directement par la fréquence.

\section{Choix du cristal acousto-optique}

L'efficacité de diffraction de ce type de modulateur dépend de la puissance acoustique mais aussi du cristal. Dans le cas d'un cristal isotrope, la résolution des modes couplés (onde incidente et onde diffractée) indique que l'efficacité de diffraction est proportionnelle à la longueur d'interaction, la puissance acoustique et le facteur de mérite $\mathrm{M}_{2}$ du matériau défini par $\mathrm{M}_{2}=\mathrm{n}^{6} \mathrm{p}^{2} / \rho \mathrm{V}^{3}$, où $\mathrm{n}$ est l'indice optique, $p$ le coefficient élasto-optique, $\rho$ la densité du matériau acousto-optique et $\mathrm{V}$ la vitesse acoustique. Le coefficient élasto-optique varie peu d'un matériau à l'autre. Le matériau acousto-optique idéal a donc un indice élevé et une vitesse acoustique aussi faible que possible. La paratellurite $\left(\mathrm{TeO}_{2}\right)$ s'est ainsi imposée comme le matériau de référence actuel. D'autres matériaux, malheureusement moins dociles, sont encore plus prometteurs, comme le Tellure ou le Calomel. La technologie actuelle de transducteur permet de générer des densités de puissance acoustique de l'ordre de $10 \mathrm{~W} / \mathrm{cm}^{2}$. Les efficacités de diffraction ainsi accessibles sont de 80 à $100 \%$.

\section{Commuter la lumière}

De telles efficacités conduisent naturellement à utiliser le modulateur acoustooptique en tant que modulateur d'intensité ou pour commuter la lumière. La vitesse de commutation dépend alors de la taille du faisceau incident et de la vitesse acoustique. Ces commutateurs utilisés dans les lasers nanosecondes Q-switchés le sont aussi dans les oscillateurs picosecondes ou femtosecondes pour faire du "cavity dumping ", c'est-àdire extraire l'impulsion de la cavité. Pour optimiser la vitesse de commutation, il faut diminuer la taille du faisceau et utiliser un matériau dont la vitesse acoustique est grande (au détriment de l'efficacité). Des temps de commutation typiques de l'ordre de 5 ns à quelques centaines de nanosecondes sont ainsi obtenus.

Comme le montre cet exemple, I'optimisation d'un modulateur acousto-optique est toujours un compromis entre de nombreux paramètres technologiques, physiques et fonctionnels.

\section{Cas des lasers femtosecondes}

\section{L'impact de la durée des impulsions}

Pour les lasers femtosecondes ultracourts (typiquement 20 fs et moins), la principale problématique est l'optimisation de la durée. La durée de ces impulsions est si brève que leur bande spectrale couvre une centaine de nanomètres autour, de $800 \mathrm{~nm}$ pour les lasers Titane Saphir. La variation de l'indice des matériaux sur une telle bande disperse l'impulsion, dont la durée n'est alors plus minimale. Des dispositifs de compensation à base de prismes ou de réseaux $\gg$

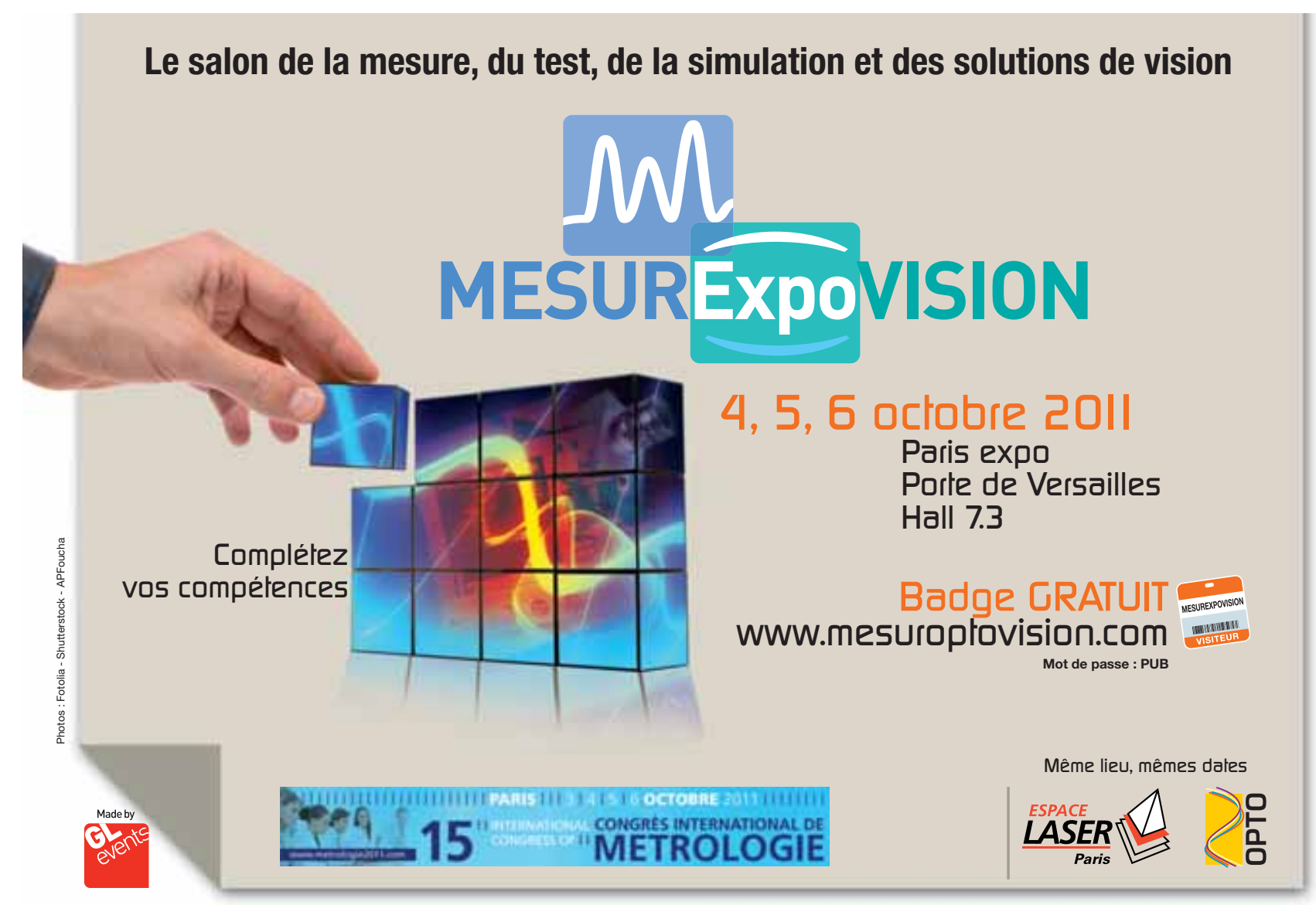


permettent de compenser partiellement cette dispersion. Ces dispositifsétirent ou compriment l'impulsion en introduisant des longueurs de chemin optique différentes suivant la longueur d'onde. Ils sont à la base de la technique d'amplification par dérive de fréquence qui, pour augmenter l'énergie des impulsions sans altérer la durée, étire d'abord l'impulsion temporellement, de quelques femtosecondes à la centaine de picosecondes, pour éviter de casser les amplificateurs. En effet, la principale limite est en puissance. L'impulsion étirée est ensuite amplifiée du nanoJoule au milliJoule, voire jusqu'à quelques Joules. Enfin, un dispositif symétrique au système d'étirement recomprime l'impulsion temporellement. Cependant la difficulté de réglage limite leur utilisation à une optimisation de la recompression aux premiers ordres du développement de Taylor de la phase spectrale.

La nécessité d'avoir un filtre linéaire est donc apparue avec la diminution de la durée (augmentation de la bande spectrale) et l'augmentation de la puissance de ces lasers. En effet, les systèmes commerciaux actuels permettent d'obtenir «en

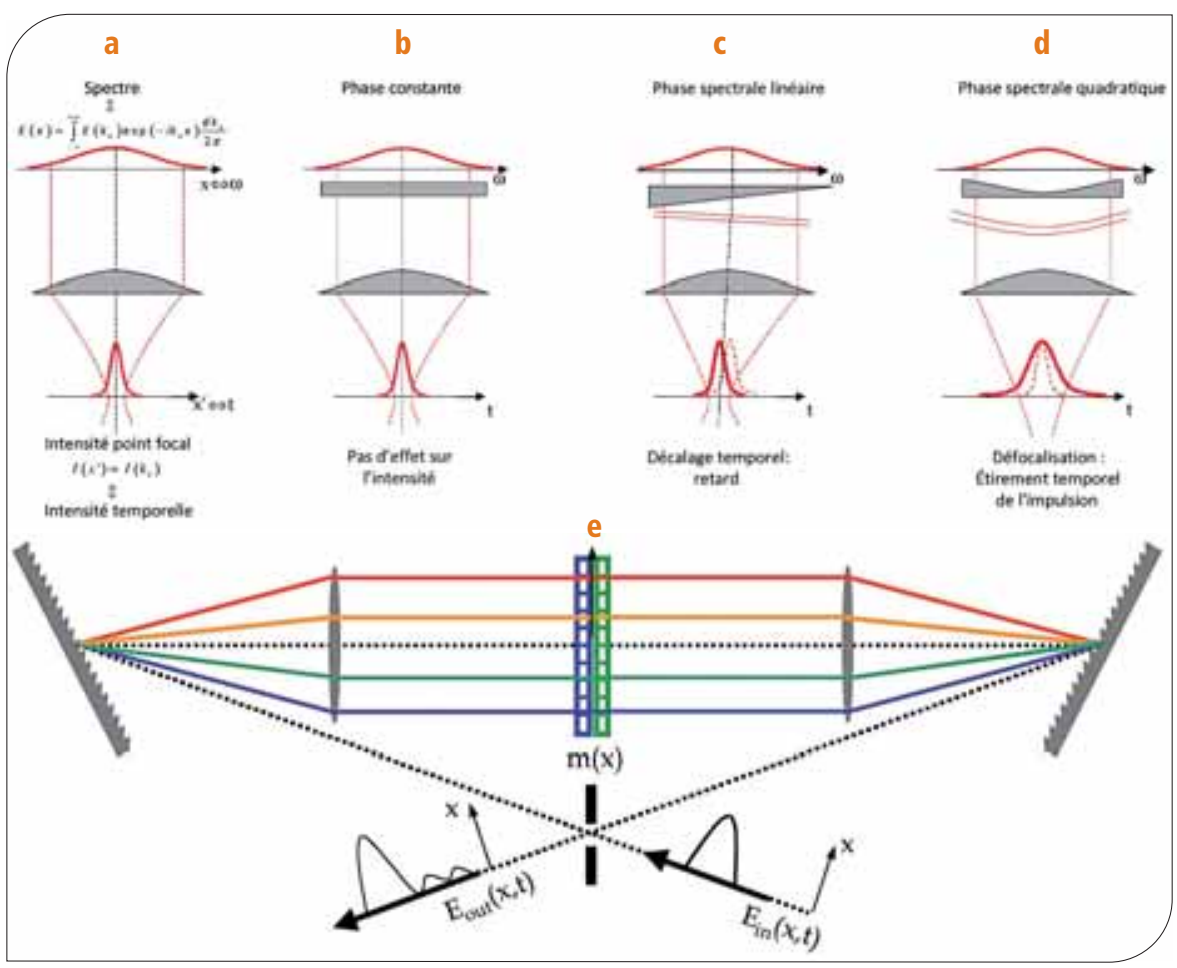

Figure 2. a à d: Analogies spatio-temporelles pour des phases nulle (a), constante (b), linéaire (c) et quadratique $(d)$; le plan avant lentille correspond au domaine spectral, le plan focal au domaine temporel. (e) : dispositif de mise en forme par ligne $4 \mathrm{f}$ à dispersion nulle. lise " l'impulsion et provoque ainsi un étalement temporel (figure $2 d$ ). Cet étalement est celui utilisé pour l'amplification à dérive de fréquence. Les ordres supérieurs distordent le profil temporel par l'intermédiaire d'aberrations.

Ainsi, si l'on couple spatialement les fréquences optiques en introduisant une phase spatiale, on introduit également une phase spectrale. Ce principe est utilisé dans le dispositif de la figure 2e, dont le montage optique, dit de ligne $4 f$ à dispersion nulle, présente un plan de Fourier dans lequel les longueurs d'onde sont spatialement étalées. Le modulateur spatial inséré dans ce plan peut alors introduire une modulation de phase et d'amplitude. Des modulateurs à base de cristaux liquides (Spatial Light Modulator) ou d'acousto-optique sont utilisés. Bien que ce dispositif soit simple à comprendre, son comportement et son optimisation requièrent de simuler convenablement le couplage spatiotemporel utilisé. De plus, la complexité d'alignement et de maintenance de ces dispositifs ainsi que leurs défauts spatiotemporels, sont contraires à l'exigence de robustesse et de fiabilité nécessaires à I'utilisation quotidienne des lasers femtosecondes de haute puissance.

\section{Dispositif acousto-optique dispersif programmable}

\section{Principe général}

Une alternative imaginée par Pierre Tournois en 1997 est d'obtenir un dispositif dispersif programmable en utilisant une cellule de Bragg de type longitudinal ou colinéaire. Le principe est alors proche des miroirs diélectriques multicouches "chirpés».

Dans le filtre acousto-optique dispersif programmable (figure 3), nommé Dazzler par Frédéric Verluise en hommage à I'héroïne de Comics, I'onde acoustique générée se propage avec une vitesse $V$ selon l'axe $z$ du cristal et reproduit donc spatialement la forme temporelle de I'onde RF. Deux modes optiques peuvent être couplés efficacement par l'interaction acousto-optique dans le cas d'un 
accord de phase. S'il y a localement une seule fréquence dans le réseau acoustique, alors une seule fréquence optique peut être diffractée en cette position. L'impulsion optique incidente est polarisée selon l'axe rapide du cristal biréfringent. Chaque fréquence optique $\omega$ pénètre le cristal sur une certaine distance avant de croiser une fréquence spatiale acoustique en accord de phase dans le réseau acoustique. À ce point $z(\omega)$, une partie de l'énergie optique est diffractée sur la polarisation de l'axe lent. L'impulsion sortant du dispositif sur la polarisation extraordinaire sera le résultat de toutes les composantes spectrales qui ont été diffractées aux différentes positions. Comme les vitesses selon ces deux axes sont différentes, chaque fréquence optique « voit » un retard optique différent exprimé par :

$\tau(\omega)=\left(z(\omega) / v_{g 1}\right)+((L-z(\omega))) / v_{g 2}$, où L est la longueur du cristal et $\mathrm{v}_{\mathrm{g} 1}$ et $\mathrm{v}_{\mathrm{g} 2}$ sont, respectivement, les vitesses de groupe des modes ordinaire et extraordinaire.

L'amplitude de l'impulsion de sortie, ou l'efficacité de diffraction, est contrôlée par la puissance acoustique à la position z $(\omega)$. L'impulsion sortant du Dazzler, $E_{\text {out }}(t)$, est une fonction de l'impulsion incidente $\mathrm{E}_{\text {in }}(\mathrm{t})$ et du signal acoustique $\mathrm{S}(\mathrm{t})$. Plus précisément, Pierre Tournois a démontré en 1997 que pour de faibles valeurs de la puissance acoustique, ce signal de sortie est proportionnel au produit de convolution du signal optique d'entrée par le signal acoustique dilaté : $E_{\text {out }}(t) \propto E_{\text {in }}(t) \otimes S(t / \alpha) \Leftrightarrow E_{\text {out }}(\omega)=$ $E_{\text {in }}(\omega) S(\alpha \omega)$, où le facteur de dilatation $\alpha$ est le rapport de la fréquence acoustique par la fréquence optique.

Dans cette formulation, le Dazzler est exactement un filtre linéaire.
Sa réponse est $S(\alpha \omega)$. Ainsi, en générant la bonne fonction, il est possible de réaliser une convolution arbitraire avec une résolution temporelle donnée par l'inverse de la bande spectrale du filtre.

Cette description qualitative illustre le principe de fonctionnement du Dazzler. Un document présentant le fonctionnement détaillé de ce modulateur acoustooptique particulier est disponible sur www.photoniques.com en «complément Internet».

\section{Applications}

La principale application de ce dispositif est la correction de l'amplitude et de la phase spectrale des impulsions ultrabrèves. Le Dazzler est alors introduit dans une chaîne femtoseconde de forte puissance (typiquement $20 \mathrm{fs}, 100 \mathrm{TW}$ ). La dimension du cristal et l'extrême puissance des impulsions optiques sortantes de ce laser interdisent de placer le cristal en sortie. Il est inséré dans la chaîne avant l'amplification. Sa dimension extrêmement compacte (inférieure à $80 \times 50 \mathrm{x}$ $20 \mathrm{~mm}^{3}$ ) facilite son implantation. Le Dazzler permet alors de compenser tous les ordres élevés et ceci sur une bande très importante. Cependant pour compenser il convient de mesurer la phase spectrale. La mesure de la phase spectrale est en soi une difficulté technique. En dehors des deux principales techniques utilisées jusqu'à présent, le Frequency-Resolved Optical Gating (inventé par Rick Trebino en 1993) et le Spectral Phase Interferometry for Direct Electric-Field Reconstruction (inventé par lan Walmsley en 1998), une nouvelle méthode introduite récemment, la Self-Referenced Spectral interferometry, permet de mesurer très précisément sur une grande dynamique

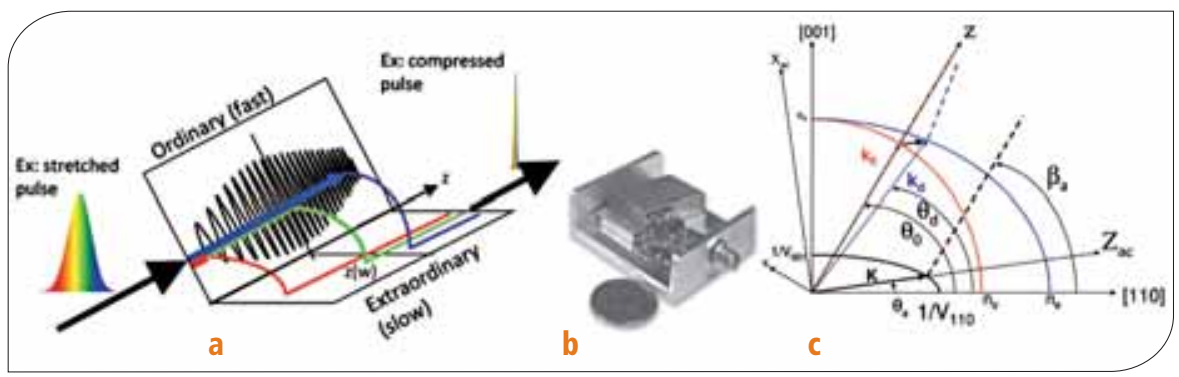

Figure 3. a. Schéma. b. Photographie du modulateur acousto-optique Dazzler. c. Diagramme des courbes de lenteurs acoustique et optique, et des vecteurs d'onde.

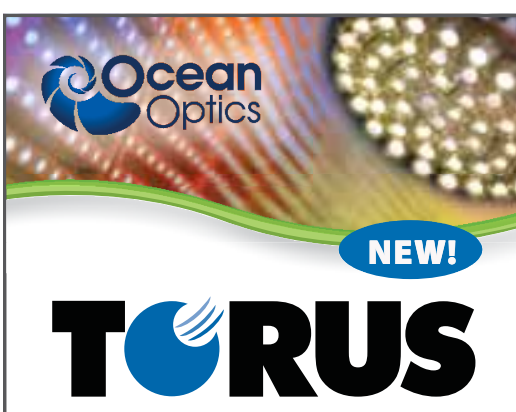

The low stray light spectrometer

The Torus is an aberration-corrected holographic concave diffraction grating spectrometer with excellent throughput and low stray light. What's more, Torus has good thermal stability - wavelength drift is mitigated and peak shape stays the same over a wide temperature range.

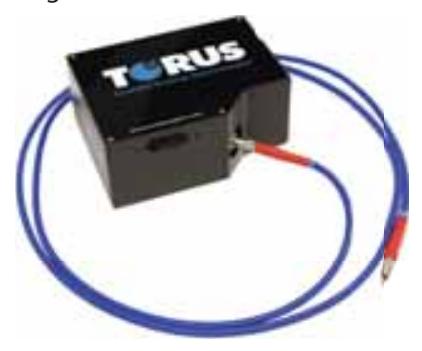

Application Range

» Precision measurement of LEDs, lasers and other light sources

» Trace gas amounts in the atmosphere

» Absorbance of optically dense solutions

» Medical dosimetry in tissue and biological media

» Fluorescence from solid surfaces, backscattering and fluorescence in solutions and powders

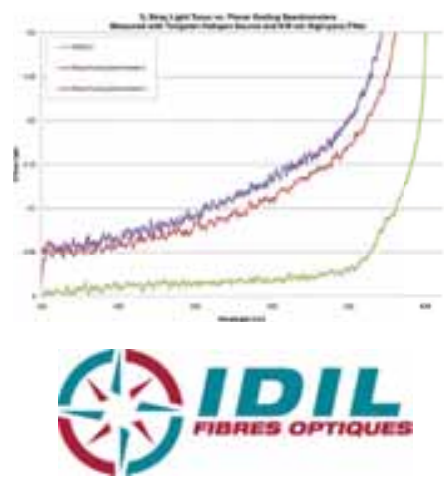

Contact us today to learn more about this new spectrometer offering:

T: 0296054020

info@idil.fr | www.idil.fr 

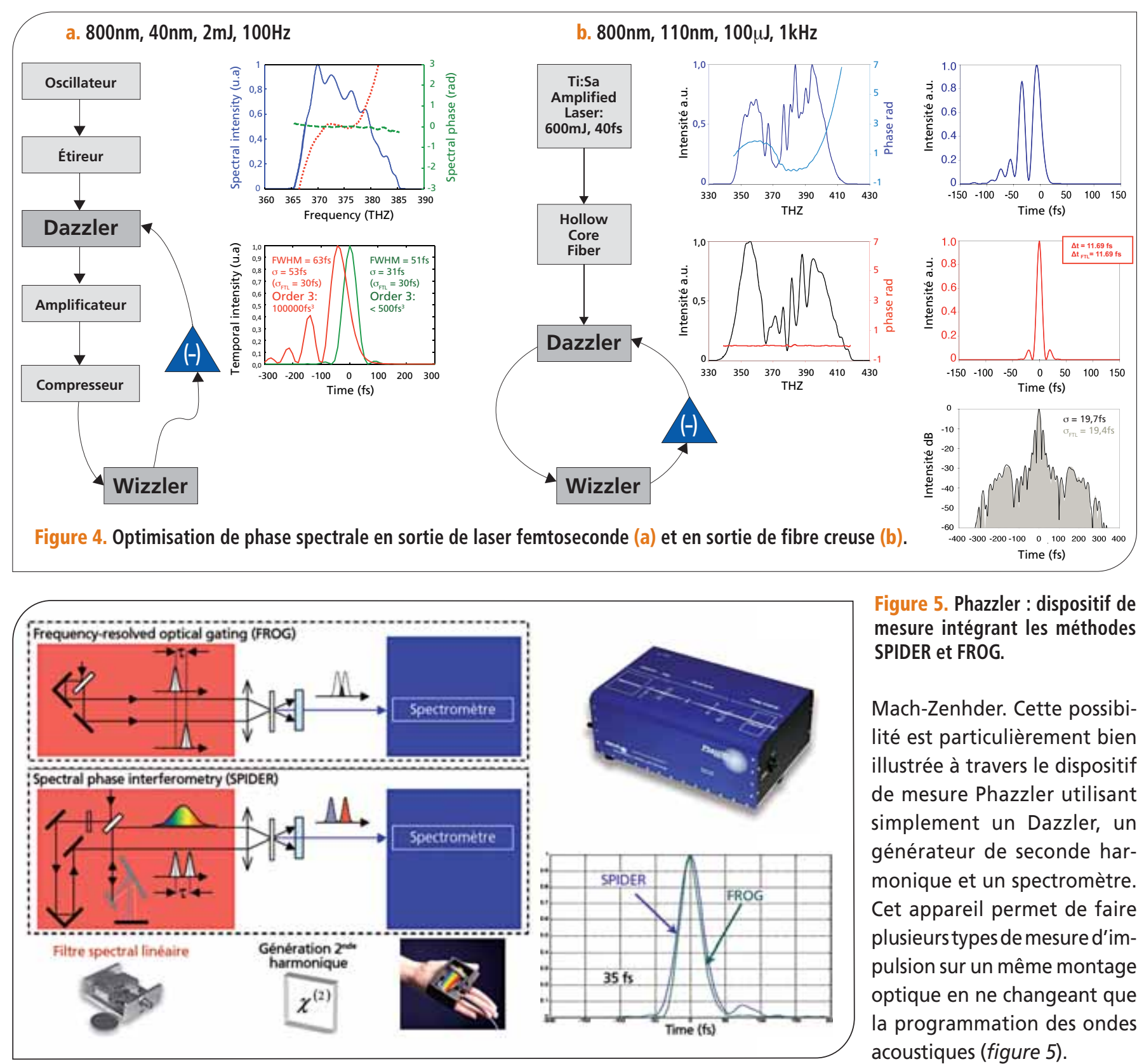

Figure 5. Phazzler : dispositif de mesure intégrant les méthodes SPIDER et FROG.

Mach-Zenhder. Cette possibilité est particulièrement bien illustrée à travers le dispositif de mesure Phazzler utilisant simplement un Dazzler, un générateur de seconde harmonique et un spectromètre. Cet appareil permet de faire plusieurs types de mesure d'impulsion sur un même montage optique en ne changeant que la programmation des ondes acoustiques (figure 5).

Cet exemple de montage

la phase et l'amplitude spectrale. La correction de l'impulsion est alors très simplement obtenue en bouclant la valeur de phase mesurée sur le Dazzler qui introduit alors la correction. Cette correction, étant seulement une perturbation comparée à la dérive de fréquence utilisée pour l'amplification, et bien que le Dazzler soit introduit avant les amplificateurs, est linéairement transmise jusqu'en sortie.

La figure 4a illustre le type de montage schématique et les résultats typiques obtenus en termes de correction. L'impulsion est parfaitement comprimée (50 fs) en sortie. De même sur des impulsions plus courtes obtenues par un élargissement spectral dans une fibre creuse (figure 4b), i lest possible de re-comprimer jusqu'à la limite de Fourier y compris sur une dynamique importante (supérieure à $40 \mathrm{~dB}$ ).

Mais le Dazzler n'est pas seulement un "optimisateur» de phase spectral mais bien un façonneur d'impulsion. Il est possible d'obtenir des profils temporels particuliers comme des impulsions carrées, paraboliques ou gaussiennes temporellement, sur une grande dynamique.

En tant que filtre linéaire, il peut simuler tout montage optique comme un interféromètre de type Michelson ou optique directement obtenu par le biais d'un Dazzler illustre tout l'intérêt d'un filtre linéaire en optique. Tout comme en électronique, il devient alors possible par des stratégies de signaux adaptés de faire du traitement du signal évolué. L'aire de l'optique femtoseconde programmable commence.

\section{Complément Internet}

《Retrouvez le fonctionnement détaillé du filtre acousto-optique programmable Dazzler sur : www.photoniques.com, rubrique La revue/ Les sommaires. $\gg$ 